\title{
Varenicline for smoking cessation: Is it a heartbreaker?
}

\author{
J. Taylor Hays MD
}

See related research article by Singh and colleagues on page 1359 and at www.cmaj.ca/lookup/doi/10.1503/cmaj.110218 and related letters on page 1404 .

Competing interests:

J. Taylor Hays has received grant funding from Pfizer to conduct a trial of varenicline.

This article was solicited and has not been peer reviewed.

Correspondence to: J. Taylor Hays, hays.taylor@mayo.edu

CMAJ 2011. DOI:10.1503 /cmaj.110804
I $\mathrm{n}$ this issue of $C M A J$, Singh and colleagues ${ }^{1}$ present a meta-analysis assessing the risk of serious adverse cardiovascular events associated with the use of varenicline for smoking cessation. The paper raises additional questions about a drug that has already come under scrutiny by the US Food and Drug Administration for neuropsychiatric safety concerns, ${ }^{2}$ and within the past month, the drug has been the focus of another warning regarding an association between it and serious adverse cardiovascular events. ${ }^{3}$ This new warning is based on observations published in a randomized trial of varenicline for the treatment of tobacco dependence among participants with known cardiovascular disease. ${ }^{4}$ Certain serious adverse cardiovascular events were seen more frequently among participants receiving varenicline than among those receiving a placebo, but the differences failed to reach statistical significance and events were rare in both treatment groups.

The concerns about the cardiovascular safety of varenicline raised by this new warning makes the meta-analysis by Singh and colleagues ${ }^{1}$ timely and important. Varenicline is efficacious for smoking cessation, ${ }^{5}$ but could this be one more case in which the treatment is worse than the condition being treated? A measured view of the evidence of the harms of smoking compared with the potential harms of varenicline treatment suggests otherwise.

In their landmark epidemiologic study of British male doctors, Doll and colleagues showed that smoking kills more than half of persistent smokers. ${ }^{6}$ In the similarly influential Nurses' Health Study (a prospective cohort study), 104000 US women were followed for 20 years, and the relative risk of mortality from coronary heart disease among women who smoked was four to five times the risk seen among women who had never smoked.? This study also showed that quitting smoking is associated with a rapid decline in risk of death due to coronary heart disease, with over $60 \%$ of the full potential benefit occurring within five years.

Given such evidence, there is no doubt that effective treatment for tobacco dependence will reduce the risk of death and morbidity related to cardiovascular disease. A considerable evidence base supported by multiple randomized controlled clinical trials and meta-analyses shows that varenicline consistently more than doubles the chances of long-term abstinence from tobacco. ${ }^{5.89}$ Thus, varenicline should be an important tool for reducing cardiovascular events among patients who smoke. How then are we to interpret and apply the results of the meta-analysis provided by Singh and colleagues ${ }^{1}$ to our clinical practices? In this regard, there are several important points to be made.

First, the main result of the meta-analysis, a $72 \%$ increased risk of serious cardiovascular adverse events, must be tempered by the rarity of these events among partipants in both treatment groups $(1.06 \%$ among patients given varenicline and $0.82 \%$ among patients given a placebo) an absolute percent difference of only $0.24 \%$.

Second, as noted by Singh and colleagues, the rate of participants lost to follow-up was greater in the placebo arm than in the treatment arm in most of the studies included in the analysis. This introduces bias in determining serious, adverse, cardiovascular events that favours fewer events counted among participants given a placebo.

Third, cardiac events were adjudicated in only a single study. ${ }^{4}$ As mentioned earlier, in that study, no significant differences were seen in the incidence of cardiovascular events or in mortality between people receiving varenicline and those receiving a placebo. ${ }^{4}$ 
Finally, although the point estimates for the number needed to treat (10) and the number needed to treat for harm (28) are similar, the degree of uncertainty for the number needed to treat for harm (upper bound of $95 \%$ confidence interval [CI 213 ) is considerably greater than it is for the number needed to treat (upper bound of 95\% CI 13). These results represent a significant degree of uncertainty about the relative good or harm from varenicline, leaving the issue unsettled. As such, how should the results of this meta-analysis guide future studies and clinical practice?

The best outcome from this analysis would be more rigorous and adequately powered studies evaluating the safety of using varenicline among smokers who have known cardiovascular disease. The worst outcome would be for health care providers to abandon the use of varenicline, which has proven to be among the most efficacious pharmacotherapies used for the treatment of tobacco dependence. ${ }^{89}$

Singh and colleagues urge clinicians to "carefully balance" the risks and benefits of varenicline. ${ }^{1}$ Although their results suggest that a measure of caution should be taken in prescribing varenicline for the treatment of tobacco dependence, the small absolute risk of cardiovascular events associated with taking varenicline is outweighed by the enormous benefit of reducing cardiovascular morbidity and mortality that can be achieved with successful abstinence from smoking.

Is varenicline a safe drug? Multiple randomized clinical trials and meta-analyses indicate that it is. ${ }^{5,89}$ Is varenicline risk free? Clearly it is not, as the meta-analysis presented by Singh and colleagues shows. ${ }^{1}$ However, the risk for serious cardiovascular adverse events is low and is greatly outweighed by the benefits of diminishing the truly "heartbreaking" effects of smoking.

\section{References}

1. Singh S, Loke YK, Spangler JG, et al. Risk of serious adverse cardiovascular events associated with varenicline: a systematic review and meta-analysis. CMAJ 2011;183;1359-66.

2. US Food and Drug Administration. The smoking cessation aids varenicline (marketed as Chantix) and bupropion (marketed as Zyban and generics): suicidal ideation and behavior. FDA Drug Safety Newsletter 2009;2:1-4

3. US Food and Drug Administration. FDA Drug Safety Communication: Chantix (varenicline) may increase the risk of certain cardiovascular adverse events in patients with cardiovascular disease. Available: www.fda.gov/drugs/drugsafety/ucm259161 .htm (accessed 2011 June 23).

4. Rigotti NA, Pipe AL, Benowitz NL, et al. Efficacy and safety of varenicline for smoking cessation in patients with cardiovascular disease: a randomized trial. Circulation 2010;121:221-9.

5. Cahill K, Stead LF, Lancaster T. Nicotine receptor partial agonists for smoking cessation. Cochrane Database Syst Rev 2011; (2):CD006103.

6. Doll R, Peto R, Boreham J, et al. Mortality in relation to smoking: 50 years' observations on male British doctors. BMJ 2004; 328:1519-33.

7. Kenfield SA, Stampfer MJ, Rosner BA, et al. Smoking and smoking cessation in relation to mortality in women. JAMA 2008;299:2037-47.

8. Eisenberg MJ, Filion KB, Yavin D, et al. Pharmacotherapies for smoking cessation: a meta-analysis of randomized controlled trials. CMAJ 2008; 179:135-44.

9. Treating tobacco use and dependence: 2008 update. Clinical practice guideline. Rockville (MD): US Department of Health and Human Services. Public Health Service; 2008.

Affiliation: J. Taylor Hays is with the Department of Medicine, Mayo Clinic, Rochester, Minn. 